\title{
EPMA EDS/WDS Comparative Analysis on Bulk and Lamella Aluminide Coatings on Stainless Steel
}

Joshua Silverstein and Bethany Matthews

Pacific Northwest National Laboratory, Richland, Washington, United States

Aluminide coatings can provide an effective hydrogen permeation barrier on 316 stainless steel claddings in nuclear environments. Analysis of these materials utilizing focus ion beam (FIB) as a sample preparation approach provides keys insights to complex microstructures on the submicron scale. Increasing probe current, probe density and accelerating voltage using microscopy techniques provides the ability to create a coherent beam of electrons to be transmitted through the sample material for electron probe microanalysis (EPMA), minimizing adsorption and secondary effects.

To prepare a tiered sample, an FEI Helios Nanolab 660 dual-beam focused ion beam (FIB) is used (1),(Fig $1)$. The interface or region of interest is capped with electron beam deposited $\mathrm{Pt}(\sim 0.5 \mu \mathrm{m})$ and ion beam deposited $\mathrm{C}(3 \mu \mathrm{m})$. A deep lamella $(25-30 \mu \mathrm{m})$ is cut and removed using a standard lift-out procedure. The lamella is attached to the tip of a $\mathrm{Cu}$ grid finger (fingers parallel to the FIB floor), which is then removed from the FIB and flipped up 90o (fingers perpendicular to the FIB floor) and returned to the FIB. The lamella is detached from that grid and attached in the flag position to a grid. The interface or region is now horizontal to the beam. The top of the lamella is coated with $3 \mu \mathrm{m}$ of ion deposited $\mathrm{C}$ to help prevent curtaining. Regions are then thinned with the ion beam at $30 \mathrm{kV}$ and $0.79 \mathrm{nA}$ by milling both sides of the lamella in sections to leave roughly $5 \mu \mathrm{m}$ wide sections of the desired thickness. When the desired thicknesses are reached, the sample is polished at $5 \mathrm{kV}$ and $81 \mathrm{pA}$ for roughly 1 minute per side.

Comparing composition maps using both EDS and WDS on bulk samples and lamella provides a quantitative approach for understanding beam/sample interactions on samples with various thickness using WDS (Fig 2). EPMA and wavelength dispersive spectroscopy (WDS) we can gather a wide range of x-ray lines even at high-energy not available when using low-kV approaches. EPMA-WDS provides an energy resolution for compositional analysis that energy dispersive spectroscopy (EDS) does not by utilizing five separate channels and selectively analyzing elements by precise quantification methods. Chemical maps generated by EPMA-WDS allow for chemical analysis of the microstructures and the microstructural relationships between the major and minor phases down to the sub-micron scale of the aluminide coatings. Analytical conditions for EDS and WDS analysis were evaluated using various accelerating voltages and beam currents, to determine the optimum conditions based on sample thickness. This work is an additional analytical approach to support previous work using EBSD techniques for understanding pre-irradiated aluminide coatings to serve as a baseline characterization for understanding microstructural changes in post-irradiated aluminide coatings (2). 

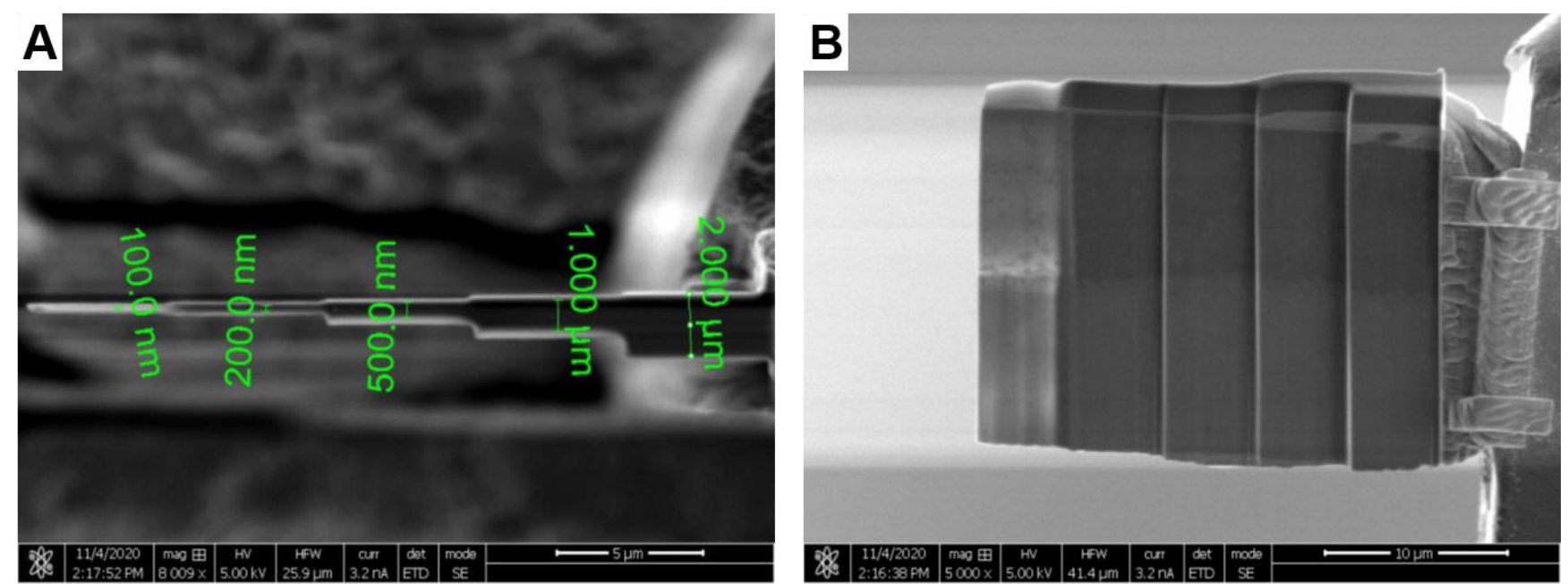

Figure 1. Micrograph of FIB prepared aluminide coating on 316 stainless steel at the interface. (A) profile and (B) top down view.
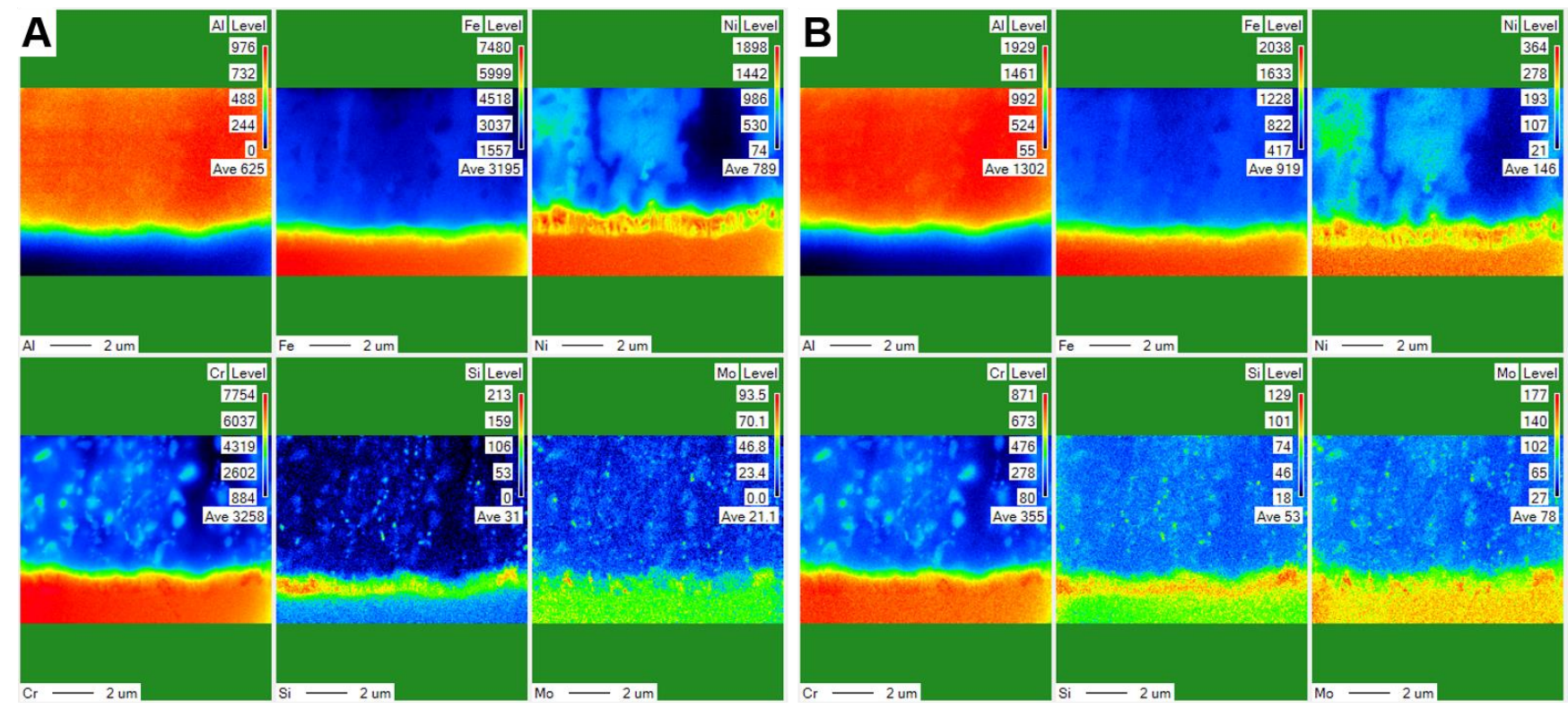

Figure 2. (A) WDS chemical map and (B) EDS chemical map collected at $30 \mathrm{kV} 20 \mathrm{nA}$ at the interface of the aluminide coating.

\section{References}

1. Boona, S. (2021). Quantitative SEM-EDS Analysis of Semi-Transparent Samples. Microscopy Today, 29(1), 42-48.

2. Johnson, B., Canfield, N., \& Matthews, B. (2019). Integrated SEM, EDS, EBSD and EPMA Characterization of Aluminide Coatings on Stainless Steel. Microscopy and Microanalysis, 25(S2), 15901591. 